\title{
Resonances and absorption enhancement in thin film silicon solar cells with periodic interface texture
}

\author{
F.-J. Haug, ${ }^{\text {a) }}$ K. Söderström, A. Naqavi, and C. Ballif \\ Ecole Polytechnique Fédérale de Lausanne (EPFL), Institute of Microengineering (IMT), Photovoltaics and \\ Thin Film Electronics Laboratory, Rue A.-L. Breguet 2, CH-2000 Neuchâtel, Switzerland
}

(Received 5 January 2011; accepted 21 February 2011; published online 22 April 2011)

\begin{abstract}
We study absorption enhancement by light scattering at periodically textured interfaces in thin film silicon solar cells. We show that the periodicity establishes resonant coupling to propagating waveguide modes. Ideally, such modes propagate in the high index silicon film where they are eventually absorbed, but waveguide modes exist also in the transparent front contact layer if the product of its refractive index and thickness exceeds half the wavelength. Taking into account that the absorption coefficient of realistic transparent conducing films exceeds the one of silicon close to its band gap, certain waveguide modes will enhance parasitic absorption in the transparent front contact. From an analysis based on the statistic distribution of energy among the available waveguide and radiation modes, we conclude that conventional thin film silicon solar cells with thick and nonideal contacts may fail to reach the previously noted bulk limit of $4 n_{\mathrm{Si}}^{2}$; instead, a more conservative limit of $4\left(n_{\mathrm{Si}}^{2}-n_{\mathrm{TCO}}^{2}\right)$ applies. (C) 2011 American Institute of Physics. [doi:10.1063/1.3569689]
\end{abstract}

\section{INTRODUCTION}

A common technological issue for silicon-based solar cells is the fact that the feasible device thickness must be kept smaller than the optical absorption length. Surprisingly, this is true for all types of silicon, regardless whether the devices are based on amorphous, ${ }^{1,2}$ nano-, micro-,, 3 or polycrystalline material; ${ }^{5}$ it even applies to wafer-based multicrystalline or single-crystal devices. ${ }^{6}$ In order to absorb sufficient amounts of light in such thin devices, light scattering at the interfaces has proven to be a highly successful concept; Redfield proposed to use surface corrugations in the range of tens of micrometers in order to refract light and ideally to trap it inside the absorber layer by total internal reflection; ${ }^{7}$ this concept has been applied ever since to wafer-based cells with typical thicknesses between 170 and 250 $\mu \mathrm{m}$, and it becomes increasingly important as the cell thickness is reduced in modern designs. Likewise, very soon after the demonstration of amorphous thin film solar cells by Carlson and Wronski, ${ }^{1}$ surface textures with accordingly reduced feature size were introduced for absorption enhancement. ${ }^{2}$

From an optical point of view, total internal reflection cannot be perfect in the presence of interface texture; light trapping must therefore be understood as an equilibrium between in- and outcoupling. Considering a slab of weakly absorbing material, Yablonovitch and Cody proposed that the ratio between effective light path and the thickness of the slab has an upper limit of $4 n^{2}, n$ being the refractive index of the absorber material. ${ }^{8}$ Besides the assumption of weak absorption and ideal Lambertian scattering at textured interfaces, the derivation of Yablonovitch and Cody also assumes a uniform distribution of energy among a continuous density of modes that take part in the coupling. Sheng et al. and Gee

\footnotetext{
a) Author to whom correspondence should be addressed. Electronic mail: franz-josef.haug@epfl.ch.
}

pointed out that modifications of the mode density by deterministic grating structures could be used to exceed the $4 n^{2}$ limit in a desired part of the spectrum at the cost of other spectral regions. ${ }^{9,10}$ By replacing continuous counting in terms of mode densities by a discrete counting scheme that takes into account coupling to waveguide modes via interface textures, Yu et al. recently reported even higher enhancement factors up to $4 \pi n^{2}$ for certain spectral regions. ${ }^{11}$ For nonsilicon absorbers with low refractive index it was proposed that embedding between high index cladding layers can enhance the absorption beyond the $4 n^{2}$ limit via coupling to evanescent fields. ${ }^{12,13}$

In this contribution, we discuss the absorption enhancement in realistic thin film solar cells based on amorphous silicon $(a-\mathrm{Si})$. Our considerations are based on the modal properties of an equivalent multilayer stack, using realistic values for the thicknesses and the refractive index dispersion of all components. The model is verified by measuring resonant absorption enhancement in reference devices manufactured on periodic grating substrates. Combining the modal structure of the realistic cell configuration with the statistical distribution of energy that is employed for the determination of light trapping limits, we conclude that in conventional devices based on silicon, the previously noted limit for light path enhancement of $4 n_{\mathrm{Si}}^{2}$ should be replaced by a more conservative value of $4\left(n_{\mathrm{Si}}^{2}-n_{\mathrm{TCO}}^{2}\right)$, taking into account the refractive index of the transparent conducting oxide, $n_{\mathrm{TCO}}$.

\section{EXPERIMENT}

We investigated light trapping in thin film silicon solar cells with periodic interface texture; in order to be fully sensitive to polarization effects, we used one-dimensional grating substrates with periods of 560 (cf. Fig. 1) and $833 \mathrm{~nm}$ and a peak-to-valley depth of $140 \mathrm{~nm}$. The grating was 


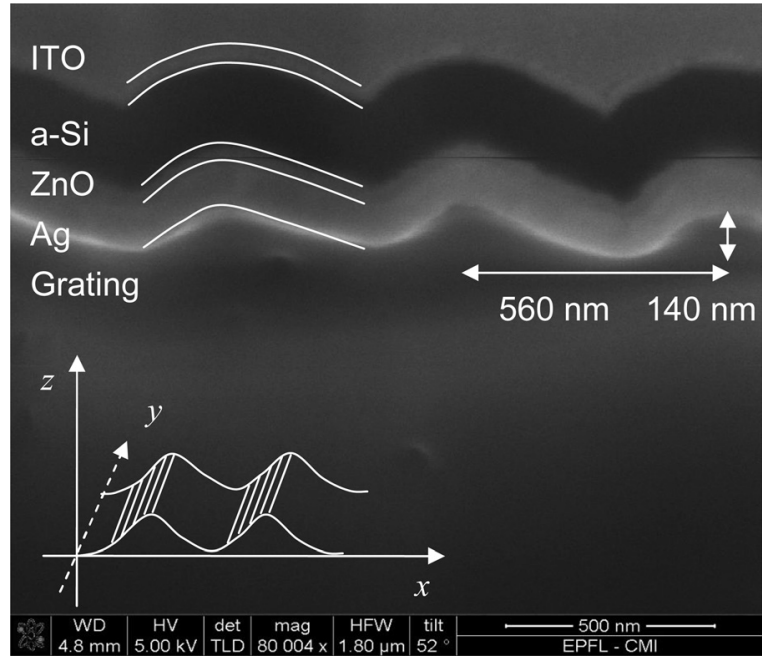

FIG. 1. Cross section through a solar cell on a line grating with period of $560 \mathrm{~nm}$ and peak to valley depth of $140 \mathrm{~nm}$. The initial blaze is gradually rounded out throughout the multilayer structure.

covered by a backreflector consisting of $120 \mathrm{~nm}$ of silver (considered semi-infinite in the theoretical section ${ }^{14}$ ) and 60 $\mathrm{nm}$ of $\mathrm{ZnO}$ buffer layer, which serves to suppress the excitation of the surface plasmon resonance (SPP) at the interface between silver and the high index silicon layer. ${ }^{15}$ The active part of the cell consists of amorphous silicon in a $n-i-p$ sequence, using a total thickness of $200 \mathrm{~nm}$, which represents a compromise between absorption and stability against light-induced degradation. ${ }^{16}$ Note that the theoretical considerations of the following sections do not take into account the minor differences in refractive index between doped and intrinsic material. Finally, a 65-nm-thick indium-tin-oxide (ITO) film serves as transparent front contact and antireflection coating. ${ }^{17}$ The cross section in Fig. 1 shows that the growth is not completely conformal, the moderate blaze of the grating substrate gets more and more rounded into a dome-like structure. Absorption enhancement was characterized by measuring the external quantum efficiency (EQE), which is the ratio of photogenerated charge carriers to the incident photon flux, measured in the spectral region between 350 and $800 \mathrm{~nm}$. The excitation of guided modes is evidenced by clearly resolved resonance peaks in the weakly absorbing region between 650 and $800 \mathrm{~nm}$. Additionally, we varied the incident angle and the polarization of the incident light. Figure 1 shows our measurement geometry; rays of incident and reflected light span the $x-z$ plane. A $1 \mathrm{D}$ grating with lines parallel to the $y$-axis thus excites guided modes that propagate parallel to the $x$-axis. Using Fresnel notation, an electric field perpendicular to the $x-z$ plane is denoted by the lower case letter "s," whereas an electric field parallel to the $x-z$ plane is associated with the lower case letter "p." In waveguide notation, the former is TE, the latter is TM. Further details can be found in Refs. 18 and 19.

\section{MODEL}

The decomposition of radiation phenomena into a superposition of discrete modes is a powerful concept; if combined with periodic boundary conditions, each discrete mode can be identified by a point in an equidistant reciprocal lattice; the same decomposition applies if the system itself is periodic in one or more directions, e.g., for gratings or crystals. In slabs, thin films, or multilayer structures, modal decomposition is still applicable when periodicity is imposed in directions parallel to the interfaces (see the following), but the treatment in the direction perpendicular to the interfaces requires more attention. $\mathrm{Yu}$ et al. employed decomposition in terms of waveguide modes. ${ }^{11}$ A particularly simple dispersion diagram is obtained for a waveguide that consists of two ideal metal plates with a separation $d$ that is filled with a material of dielectric constant $n$ because electromagnetic waves will have nodes at the metal surfaces and propagate along the dielectric. In the remainder of this contribution, we will refer to this situation as the "idealized model." If we orient the plates parallel to the $x-y$ plane, we can choose one of the axes parallel with the propagation direction, e.g., the $x$-axis. The requirement of fitting $m$ multiples of $\lambda / 2$ into the thickness of the dielectric constrains the wave vector component in the $z$-direction, resulting in the following dispersion relation: ${ }^{20}$

$$
\omega=\frac{c}{n} \sqrt{k_{\|}^{2}+k_{z}^{2}}=\frac{c}{n} \sqrt{k_{\|}^{2}+(m \pi / d)^{2}}, \quad m=1,2,3, \ldots
$$

Figure 2 shows plots of the dispersion relations for the lowest order modes, assuming a 200-nm-thick dielectric, using the realistic refractive index dispersion of amorphous silicon. In Fig. 2, it is sufficient to show only the first quadrant representing forward traveling waves because the dispersion diagram obeys mirror symmetry with respect to the vertical axis. Thus, the shown dispersion relations have equivalent branches of backward traveling modes in the second quadrant. Setting $\mathrm{k}_{\|}=0$ defines the cutoff condition of minimum energies below which no propagation is allowed. Directly above cutoff, the group velocity $d \omega / d \mathrm{k}_{\|}$is zero for all modes. When the waveguide is regarded simplistically as a set of two parallel mirrors, this situation corresponds to standing

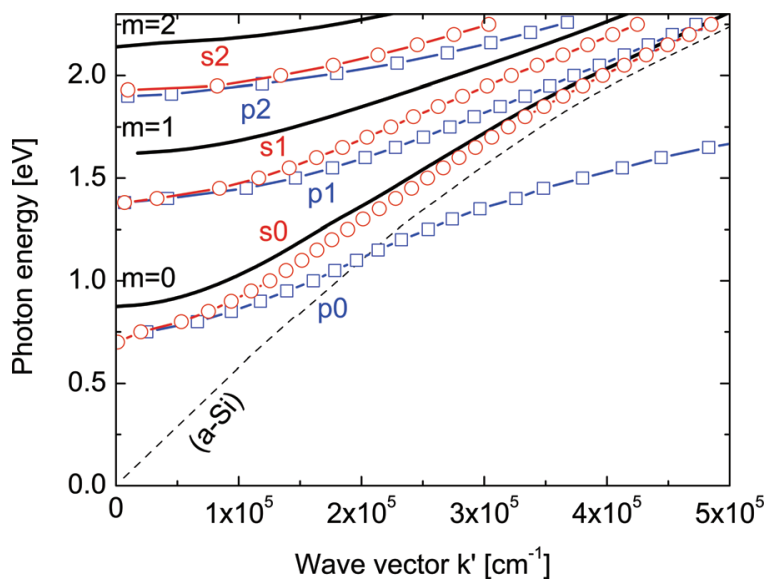

FIG. 2. (Color online) Dispersion relation of a planar waveguide consisting of a high index dielectric guiding medium (200 nm $a$-Si:H) delimited by metal on both sides. Full lines represent an ideal metal according to Eq. (1), squares and circles illustrate $p$ - and $s$-polarization for silver, respectively. The dashed line denotes the dispersion relation of light in silicon $\omega=c k_{\|}^{\prime} / n$. 
waves that bounce back and forth in the $z$-direction without propagation. With increasing energy, the dispersion relations approach the properties of wave propagation according to the dispersion relation of photons in a dielectric material $\omega=(c / n) \mathrm{k}_{\|}$. In the simplistic view, this means that the guided wave propagates parallel to the interface without feeling any confinement.

\section{WAVEGUIDE DISPERSION RELATIONS}

Analytic forms of the dispersion relation can be given only for simple situations; for realistic combinations of materials and stacks of films, they are normally determined numerically. One such method is based on the idea that propagating modes are resonances of a given system. The dispersion relations can then be identified with poles of the Fresnel coefficients, i.e., setting the denominator equal to zero and solving for $\mathrm{k}_{\|}=\mathrm{k}_{\|}^{\prime}+\mathrm{ik}_{\|}^{\prime \prime}$ at given energy $\omega .{ }^{19,21}$

Figure 2 includes the dispersion relation for the same waveguide configuration, but using real dielectric data of silver for the metal plates. Compared to the ideal modal structure of Eq. (1), the dispersion relations are split into two branches representing the two polarization directions. The splitting vanishes at the onset and asymptotically at high energies, which can again be understood within the simplistic model because at zero degrees, as well as at grazing incidence, the reflection does no longer depend on polarization. Figure 2 also shows that all modes set in at energies below those predicted by Eq. (1) because the electromagnetic fields penetrate to a finite extent into the metal boundaries, which increases the effective waveguide thickness. For silver, the photonic skin depth is between 3 and $5 \mathrm{~nm}$ in the shown energy range. ${ }^{20}$ Generally the $s$-modes are described well by Eq. (1) if the waveguide thickness $d$ is increased by about 50 $\mathrm{nm}$, but the behavior of the $p$-modes is rather difficult to match. Within the $p$-modes, the $p_{0}$ mode is special because it intersects with the silicon light line and runs at energies significantly below all other guided modes. It can be identified with a SPP whose field amplitude peaks at the dielectricmetal interface.

Figure 3 shows the dispersion relation of a solar cell stack using the dimensions given in the experimental section, and taking into account the dispersion of the refractive indices in all layers. In the remainder of this contribution, we will refer to this situation as the "realistic model." Compared to the strong confinement of the idealized metal-dielectricmetal waveguide of Fig. 2, the solar cell is characterized by much less confined fields that extend a considerable distance into the air. Some agreement between the shown modes and characteristics according to Eq. (1) can only be achieved for effective waveguide thicknesses $d_{\text {eff }}$ close to $300 \mathrm{~nm}$ instead of $200 \mathrm{~nm}$. A second difference to the configurations of Fig. 2 is the region above the light line of air, which represents a continuum of radiating modes that are not guided. In a dispersion diagram like Fig. 3, radiating modes correspond to straight lines according to the linear relation $\omega=c k$. They emerge from the origin, and only the in-plane wave vector $k_{\|}$ is considered, thus their slope can vary between vertical (along the energy axis) for perpendicular incidence and the

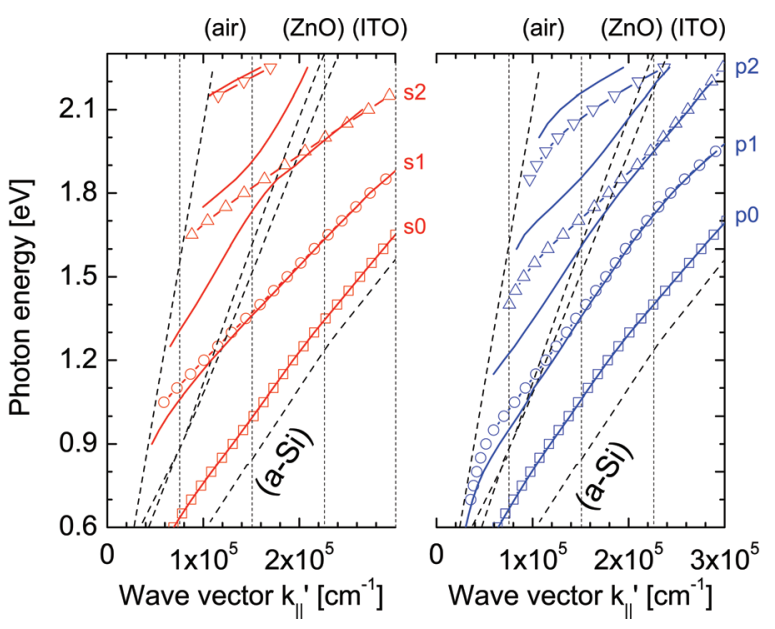

FIG. 3. (Color online) Dispersion relations of the lowest order modes of a thin film silicon solar cell represented by a stack of air/ITO $/ a-\mathrm{Si} / \mathrm{ZnO} / \mathrm{Ag}$ (symbols). The left- and right-hand panels show $s$ - and $p$-polarization, respectively. Dashed lines represent the light lines in air, ZnO ITO, and silicon. Solid lines represent the dispersions relations for tripled ITO thickness, vertical lines illustrate the Brillouin zones for a periodicity of $560 \mathrm{~nm}$ along the $x$-direction.

speed of light (denoted by the light line of air) for grazing incidence above the surface. Due to the conservation of energy and momentum, the radiation modes cannot couple to guided modes; in the dispersion diagram this is manifested by the fact that the curves of the two types of modes never intersect.

Figure 3 also illustrates the changes in the modal structure when the thickness of the ITO front contact is increased from 65 to $210 \mathrm{~nm}$. This latter thickness yields the thirdorder antireflection condition for an incident light of $550 \mathrm{~nm}$; it is sometimes chosen when $65 \mathrm{~nm}$ of ITO are not sufficient for carrying the photocurrent, and other methods of enhancing the conductivity like metallic grids or narrower interconnection stripes cannot be applied. It is observed that the modal structure between the light lines of the low index materials ( $\mathrm{ITO} / \mathrm{ZnO}$ ) and the one of silicon remain almost unchanged, but the region between the ITO/ZnO light lines and the one of air now accommodate an additional mode in the same energy range. By making the ITO layer thicker yet, this effect can be pronounced to the extent that the modal structure resembles two completely isolated waveguides; the first one is formed by an ITO guiding layer embedded between air and semi-infinite silicon and the second one corresponds to a silicon guiding layer between the low index oxides. $^{22}$ Incidentally, the same effect is observed when the thickness of the $\mathrm{ZnO}$ buffer layer is increased because the refractive indices of ITO and $\mathrm{ZnO}$ are similar.

\section{WAVEGUIDE ATTENUATION}

The imaginary part $k_{\|}^{\prime \prime}$ of the dispersion relation represents the attenuation of a given guided mode. Figure 4 compares the behavior of $k_{\|}^{\prime \prime}$ for the various modes and the two different thicknesses of the ITO front contact. At low energies, all modes follow roughly the absorption coefficient of the $\mathrm{ZnO}$ buffer layer, the only exception from this behavior is the very weakly attenuated $s_{0}$ mode. Starting from about 

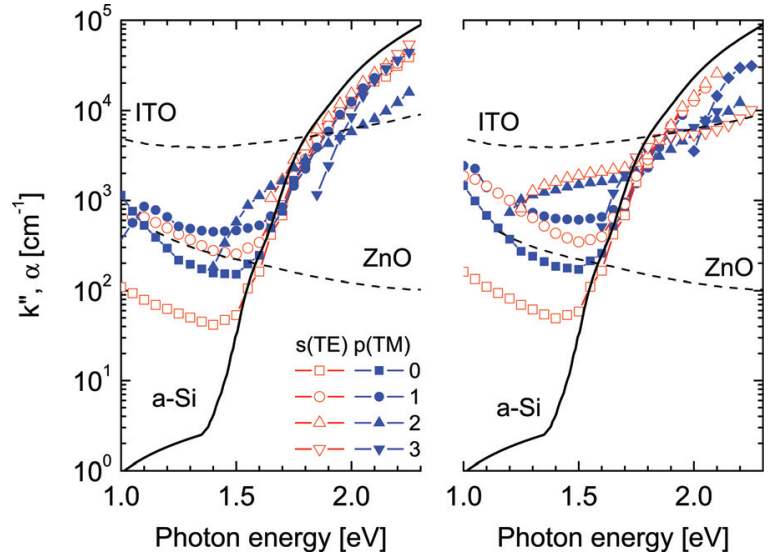

FIG. 4. (Color online) Attenuation $k_{\|}^{\prime \prime}$ of $s$ - and $p$-polarized modes from Fig. 3 ; ITO thicknesses of 65 and $210 \mathrm{~nm}$ are shown in the left- and right-hand panels, respectively. The full and dashed lines are the absorption coefficients of amorphous silicon, ITO, and $\mathrm{ZnO}$.

$1.5 \mathrm{eV}$ where $\mathrm{ZnO}$ and $a$-Si absorb equally, the attenuation of most modes starts following the absorption characteristics of the amorphous silicon absorber layer. For the case of thin ITO, only the higher order $p$-modes do not reproduce this trend, rather they deviate toward the absorption coefficient of ITO. This effect becomes more pronounced for the case of thicker ITO, and here also the highest order $s$-mode follows. Nevertheless, throughout most of the light trapping region between 1.6 and $2 \mathrm{eV}$, the numerical values of the waveguide attenuation are equal to half the absorption coefficient of silicon $\left(k_{\|}^{\prime \prime}=\alpha_{\mathrm{Si}} / 2\right)$. Waveguide attenuation itself can be related to the half-width (HWHM) of a Lorentzian resonance curve in terms of energy via $k_{\|}^{\prime \prime}=(n / 2 c) \Delta \omega .^{23}$ The combination of these two aspects yields a relation for the spectral width that guided mode resonances contribute to an absorption spectrum:

$$
\Delta \omega=\frac{c}{n} \alpha_{\mathrm{Si}}
$$

Based on temporal coupled mode theory, Yu et al. recently gave the same relation, ${ }^{11}$ but we note from Fig. 4 that it does not necessarily apply in general; depending on energy, the combination of materials and their respective thicknesses, a given mode may be dominated by absorption in ITO. Restricting ourselves to the modes that are dominated by silicon, we can expect from Eq. (2) that resonances at 1.65 and $1.75 \mathrm{eV}$ are 0.01 and $0.1 \mathrm{eV}$ wide, respectively. However, at $1.95 \mathrm{eV}$, where amorphous silicon is still considered weakly absorbing, the spectral width of the resonances is around 0.5 $\mathrm{eV}$, which would already cover the whole light trapping region.

\section{PERIODICITY AND RECIPROCAL SPACE}

Coupling between external radiation and guided modes can be achieved principally by three means: end-fired waveguides, ${ }^{24}$ frustrated total internal reflection from a prism across an air-gap, ${ }^{25,26}$ and coupling by textured interfaces. $^{27,28}$ Only the last one is relevant to solar cells because it is applicable on large areas. For the special case of a periodic texture, Brillouin zones allow a very elegant description of coupling phenomena. Figure 3 illustrates the Brillouin zones for the periodicity of $560 \mathrm{~nm}$. In the dispersion diagram, incident light is no longer uniquely defined by light lines emerging from the origin, but it can be represented by a multitude of equivalent lines that are shifted horizontally by integer multiples of the reciprocal lattice vector. Thus, coupling takes place whenever one of the equivalent light lines intersects with the dispersion relation of a guided mode in a higher order Brillouin zone. Alternatively, higher order Brillouin zones can be reduced into the first zone as illustrated in the upcoming Fig. 6.

\section{RESONANCE EXCITATION IN PERIODIC DEVICES}

After the theoretical considerations of the previous sections, we present experimental results on absorption enhancement by guided mode excitation in solar cells on periodic backreflectors. Figure 5 shows angle resolved EQE data of a cell on a grating with period of $833 \mathrm{~nm}$, corresponding data for the $560 \mathrm{~nm}$ grating can be found in Refs. 18 and 29 . Figure 5 shows that the resonance condition for the excitation of a waveguide exhibits a strong angular dispersion, whereas the interference condition at $610 \mathrm{~nm}$ is almost unaffected by the variation of the incident angle between $0^{\circ}$ and $55^{\circ}$. By noting the energy of a given resonance and converting the incident angle into a corresponding in-plane wave vector, we can determine the band structure of a real device from the experiment. Figures 6 and 7 compare the experimentally measured band structure with the theoretical results, using a reduced representation in the first Brillouin zone. We show only the forward propagating modes because the theoretical model obeys strict mirror symmetry with respect to the vertical axis. Although some asymmetry might be expected from the experimental data because of a small blaze in the grating, we note that this would primarily affect the coupling strength into forward or backward traveling, but

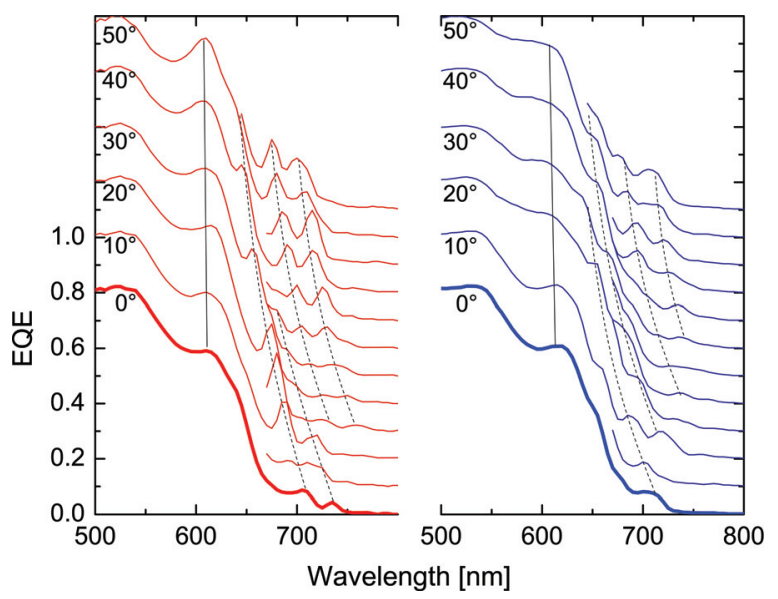

FIG. 5. (Color online) Angle resolved EQEs of solar cells on a grating with period of $833 \mathrm{~nm}$. The left- and right-hand panels show $s$ - and $p$-polarization, respectively. The scale applies to the $0^{\circ}$ measurement only (thick line), incident angles in multiples of $10^{\circ}$ are shifted vertically, intermediate characteristic are plotted only partially. The vertical line illustrates the position of an interference maximum, dashed lines illustrate positions guided mode resonances. 


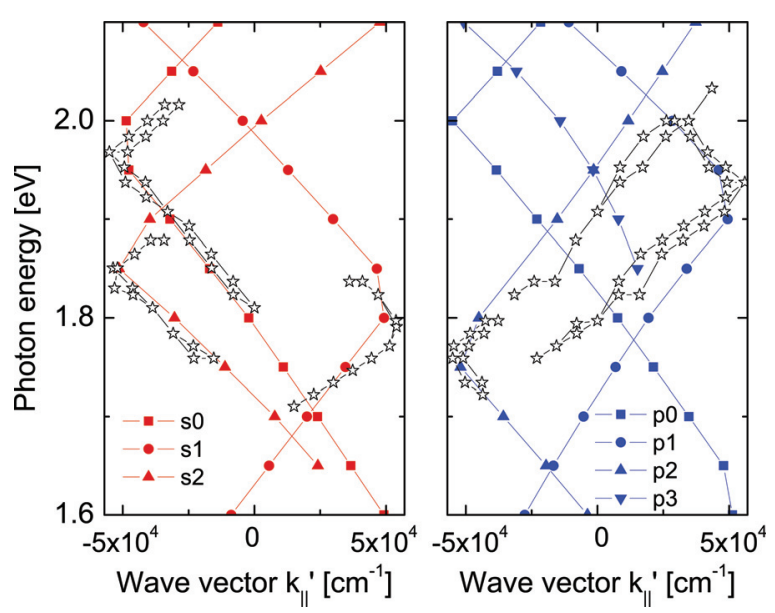

FIG. 6. (Color online) Measured dispersion diagrams after reduction into the first Brillouin zone. The left- and right-hand panels show $s$ - and $p$-polarization, respectively. Open stars denote resonances that have been measured by angle and polarization resolved EQE on a cell with $560 \mathrm{~nm}$ periodicity, small symbols reproduce the numerical modal structure of Fig. 3.

not the resonance frequencies that are governed by the periodicity.

From Fig. 6, it can be concluded that the individual modes of $s$-polarization have preferred energy ranges where they are excited; at low energies between 1.7 and $1.85 \mathrm{eV}$, we measured primarily the $s_{1}$ and $s_{2}$ modes, whereas excitation of the $s_{0}$ mode only starts at $1.8 \mathrm{eV}$ and extends beyond $2 \mathrm{eV}$. Overall, the agreement between the flat waveguide model and the experimental data on the grating is quite acceptable. Excitation of the $p$-modes proceeds rather differently; first of all, we note more deviations between the theory and the experiment, good agreement is only observed close to the Brillouin zone boundaries. Additionally, the excitation is limited exclusively to the $p_{1}$ and $p_{2}$ modes, we did not observe any coupling to the $p_{0}$ and the $p_{3}$ modes. It appears that different modes are excited on the grating with the larger period; as shown in Fig. 7, we observe that in $s$-polarization the $s_{0}$ mode is no longer excited, unfortunately in $p$-polarization a unique attribution is not possible due to overlapping characteristics for this particular choice of period and absorber thickness.
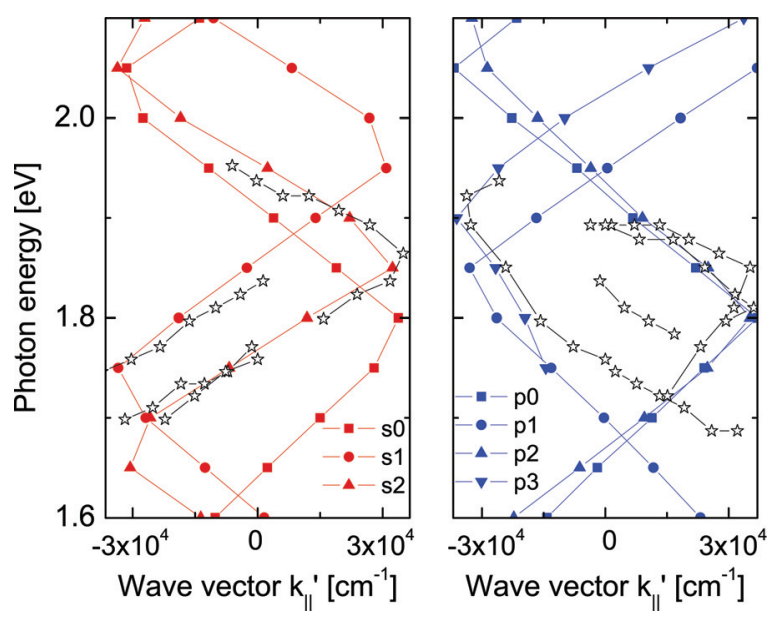

FIG. 7. (Color online) Same as Fig. 6, but measured on a grating with periodicity of $833 \mathrm{~nm}$.
Generally, we note from Figs. 6 and 7 that our theoretical model with flat interfaces describes the guided modes structure relatively well in $s$-polarization, but the correspondence is less satisfactory for $p$-polarization. In one instance (righthand panel of Fig. 7) none of the theoretical modes matched to an observed series of resonances, and no better agreement could be obtained by changing the thickness of the guiding layer within the experimental tolerances.

From the experiment it appears rather unsystematic whether a given mode gets excited or not, and simple rules for predicting such excitations or their coupling strength do not exist. For gratings with mirror symmetry, it was proposed that normally incident light should not couple to odd orders. ${ }^{11}$ This might seem contradictory to the shown experiments where even and odd orders get excited, however, note that Ref. 11 considers symmetry with respect to the $x$-direction, whereas the order of guided modes refers to their symmetry along the $z$-direction. Moreover, it is difficult to classify our experimental situation; Fig. 1 shows that the weak asymmetry of the grating substrate is quickly smoothened out into a rather symmetric dome-like shape toward the front interface. In this case, the only way to assess absorption enhancement is by rigorous numerical solution of Maxwell's equations for the multilayer structure, taking into account the exact nature of all interfaces. Based on such techniques, significant absorption enhancement has been predicted for binary and ideally blazed gratings; ${ }^{29-31}$ tailoring of surface textures for solar cells is still a rather young field. ${ }^{32}$ Moreover, not all theoretically imaginable shapes are also feasible for solar cell processing. Figure 1 illustrates that pointed structures will become rounded, whereas valleys tend to develop a deep pinch, which is undesirable in terms of electric performance. ${ }^{33}$ The conformality of the growth processes is not sufficient to manufacture exactly reproduced interfaces that underlie most of the numeric solutions, but in general it also prevents the combination of flat and textured interfaces if any such combinations were desired.

\section{A NOTE ON STATISTICAL COUPLED MODE THEORY}

Theoretical predictions of the light path enhancement assume a statistical distribution of the incident light intensity between loss channels (reflection and transmission) and absorption, either in terms of continuous modal densities (Ref. 8 ), or by taking into account a combination of discrete diffraction orders and waveguide modes (Refs. 11 and 12). The latter approach identifies the waveguide modes with a reciprocal coordinate along the $z$-direction; thus, the definition of Eq. (1) leads to an equidistant grid with spacing of $\pi / d$. Except for the restriction to positive orders, this is similar to the definition of the reciprocal space in the $x$-direction in multiples of $2 \pi / L$, where $L$ is the periodicity. Note that the extension of the $k_{z^{-}}$ coordinate toward negative mode numbers in Ref. 11 is compensated by doubling the spacing of the lattice points to $2 \pi / d$, thus introducing a small error by also counting trivial orders with $m=0$ along the horizontal axis.

We used Eq. (1) and our numerical mode structures for a conversion of the dispersion diagram into $k$-space as shown 


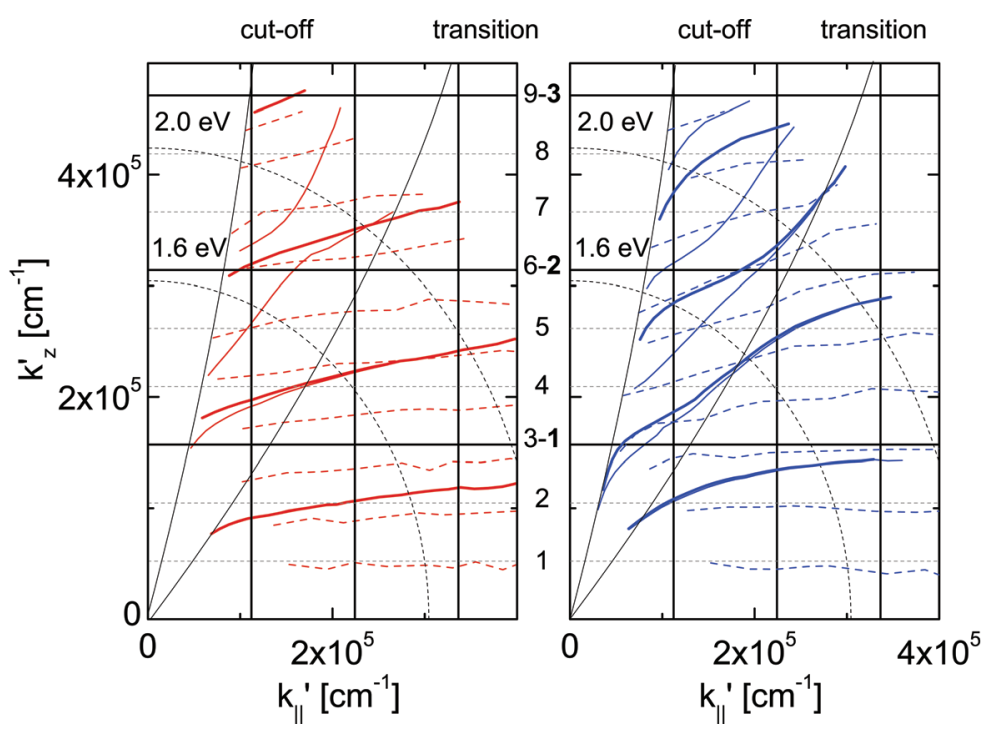

FIG. 8. (Color online) Illustration of reciprocal space distortion for $s$ - (left-hand side) and $p$-polarization (right-hand side); thick, thin, and dashed lines represent the standard cell, a cell with thick ITO $(210 \mathrm{~nm})$, and a cell with thick i-layer $(600 \mathrm{~nm})$, respectively. The rectangular grid illustrates the periodicity of a grating with $L=560 \mathrm{~nm}$ (vertical lines) and the modes related to the idealized waveguide (horizontal lines). The separation is $\pi / d$, using $d$ equal to 200 (full) and $600 \mathrm{~nm}$ (dashed), numbers between the panels give the mode number. Circle sectors illustrate the energy range of light trapping between 1.6 and $2.0 \mathrm{eV}$. in Fig. 8. The light trapping region corresponds to the area between the two circle sectors that represent light with 1.6 and $2.0 \mathrm{eV}$. Figure 8 illustrates that the changes in the effective waveguide thickness with mode number and energy distort the lattice toward a higher density of lattice points, particularly in the region of low $k_{\|}^{\prime}$. The modes due to $s$ - and $p$-polarization approach the theoretical grid only asymptotically, resulting in a distortion that is quite significant for a thin absorber layer of $200 \mathrm{~nm}$, and still noticeable when the absorber thickness is increased to $600 \mathrm{~nm}$. Because the distortion is an inherent consequence of the chosen combination of materials and their respective thickness, it must be determined for each case. As further difference to the ideal model, the cutoff condition of the real waveguide defines a region around the $k_{z}$-axis where no coupling to waveguide modes takes place. Note that we may still observe a certain amount of absorption enhancement by interference effects in this region; counting these instances as modes may therefore be justified to a certain extent.

We finish this section by comparing different procedures of the statistical analysis of the $1 \mathrm{D}$ periodic cell configurations with thin ITO $(65 \mathrm{~nm})$. We follow the procedure of Ref. 11 , but we maintain the discrete nature of the $k$-space grids shown in Fig. 8. As only simplification to the counting procedure, we use a uniform energy interval $\Delta \omega$ of $0.1 \mathrm{eV}$. If $N$ is the number of propagating diffraction orders outside of the solar cell structure at an energy $\omega$ and $\Delta M$ is the number of grid points in the distorted $k$-space in the energy interval between $\omega$ and $\omega+\Delta \omega$, then the enhancement factor $F$ for 1D periodicity is given by Eq. (7) of Ref. 11:

$$
F=\frac{2 \pi c}{n d \Delta \omega} \frac{\Delta M}{N} .
$$

Figure 9 compares $F$ for two different counting schemes that were applied to the case of the 200-nm-thick silicon layer and two different grating periods. As before, low energies are included for illustration only; while reflection measurements clearly show enhanced parasitic absorption in this region, ${ }^{19}$ it is not very meaningful from a solar cell perspective to define absorption enhancement for energies below the band gap of the absorber layer. Moreover, the number of interactions is clearly too small to justify a statistic treatment. Despite these shortcomings in terms of experimental applicability, it is still possible to compare the models among themselves, and to judge in which spectral region their use is justified; the realistic model ranges considerably below the model based on a rectangular $k$-space according to Eq. (1), and also below the continuous model. We attribute this observation to the fact that the former case counts neither trivial resonances along the axis of $k_{\|}$, nor those to the left of the cutoff region.

We turn to a case where the statistic approach becomes more applicable, i.e., an absorber layer thickness of 600 $\mathrm{nm}$. Although this is too thick for current state-of-the-art solar cells based on amorphous silicon, it is among the range of acceptable thicknesses for microcrystalline bottom cells in a recent generation of thin tandem cells that are

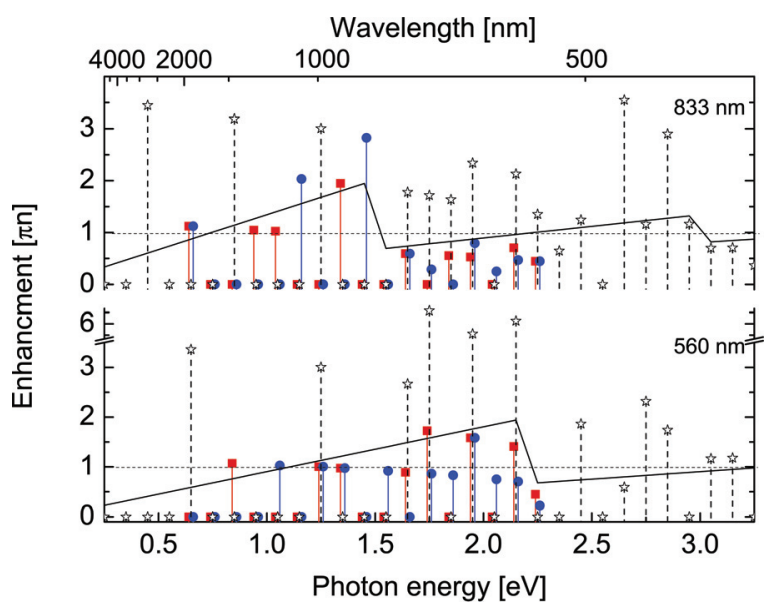

FIG. 9. (Color online) Statistical determination of the absorption enhancement for a cell stack with a 200 -nm-thick absorber layer on gratings with two different periodicities. Stars denote the idealized waveguide, squares and circles represent $s$ - and $p$-polarized modes of the realistic waveguide, respectively. The saw-tooth line shows the limiting behavior for semicontinuous mode density. Note that the small number of interactions makes this plot merely illustrative, a statistically more relevant case is shown in Fig. 10 for the case of a thicker absorber layer. 


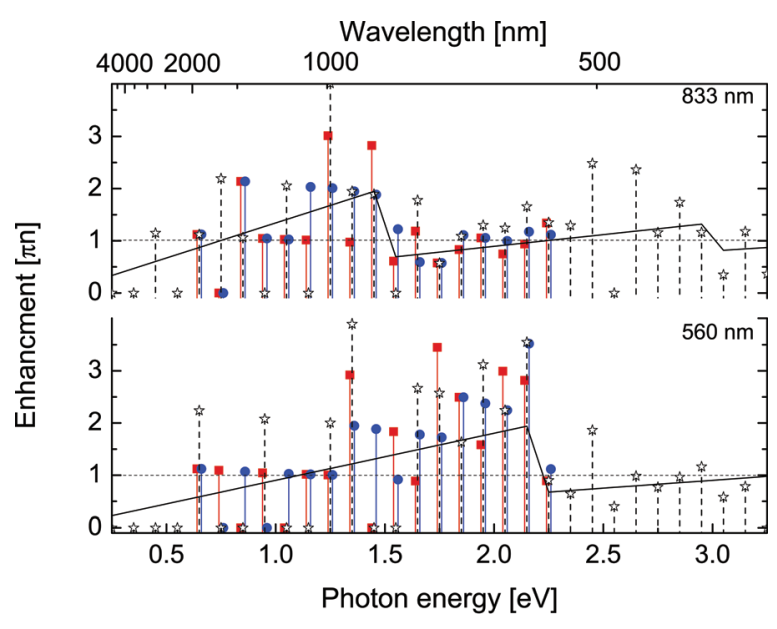

FIG. 10. (Color online) Enhancement factor for a cell stack on gratings with two different periodicities using a 600-nm-thick absorber layer. Lines and symbols have the same meaning as in Fig. 9.

attractive because of high production throughput. ${ }^{34}$ Keeping in mind the small differences in refractive index between amorphous and microcrystalline silicon, the results shown in Fig. 10 are very close to the properties of the latter cell type, and the higher number of resonances in the thicker absorber layer makes the statistical treatment more justified. In Fig. 10, the data of realistic and idealized cells are closer, but a lower average for realistic counting is nevertheless distinguishable within the fluctuations. In favor of the idealized model, we may conclude that the remaining distortion toward a higher density of resonances in the realistic description may almost compensate the counting errors of the idealized waveguide with its rectangular grid. Both discrete models exhibit clearly visible step changes at wavelengths corresponding to the grating period or integer fractions thereof. These are related to sudden changes in the number of external radiation modes by the onset of higher diffraction orders. Their occurrence shows that radiation losses must be treated with discrete counting. Compared to the data in Fig. 9, Fig. 10 shows that the fluctuations are much reduced in the discrete data of the 600$\mathrm{nm}$-thick absorber layer; the points approach already very closely to the saw-tooth-like characteristic that assumes a continuous distribution of the resonances within the waveguide, but keeps the discrete nature of the radiation modes.

Note that exactly this aspect, i.e., the discrete nature of the loss channels gives rise to absorption enhancement beyond previously reported limits; ${ }^{11}$ instead of $\pi n$, a $1 \mathrm{D}$ case may exhibit enhancement factors $F_{1 \mathrm{D}}$ of up to $2 \pi n$ at energies just below (i.e. wavelengths just above) the onset of the first order diffraction modes, likewise, on a 2D grating $F_{2 \mathrm{D}}$ can be as high as $4 \pi n^{2}$ instead of the $4 n^{2}$ limit of Yablonovitch and Cody.

\section{LIGHT PATH ENHANCEMENT IN CELLS CONFIGURATIONS WITH THICK TRANSPARENT CONDUCTING OXIDES}

In this section we would like to discuss a second aspect of the distortion of $k$-space shown in Fig. 8. Among the samples with different ITO thicknesses, the one with the thicker layer shows a higher density of modes directly after cut-off, but almost unchanged characteristic for high $k_{\|}$values. Figure 4 reveals that the attenuation of these modes is largely dominated by the absorption of ITO, not silicon. As mentioned earlier, the same effect is observed for thicker $\mathrm{ZnO}$ buffer layers, and applies therefore also to cell designs with thick front contacts, e.g., cells using textured $\mathrm{SnO}_{2}: \mathrm{F}$ or $\mathrm{ZnO}$ contacts with thickness between 600 and $2000 \mathrm{~nm}^{16,35}$ In these cases, the low index layers are thick enough to support their own guided modes, resulting in three clearly defined regimes in $k$-space; a continuum of "air-like" modes to the right of the cutoff, "TCO-like" modes (for transparent conducting oxide) between cutoff and a transitional region, and "silicon-like" modes to the right of this transition. Because absorption in realistic contact materials is comparable to, if not higher than, silicon throughout the light trapping region (cf. Fig. 4), it is no longer appropriate to consider all interactions in the energy interval between $\omega$ and $\omega+\Delta \omega$. Instead, for cells with thick contact layers, statistics should be extended only over interactions to the right of the "transition" in Fig. 8. Equivalently, counting of interactions can be carried out in a nonreduced dispersion diagram like Fig. 3, considering only interactions between the light lines of TCO and silicon.

To illustrate this point, Fig. 11 compares the dispersion diagrams a free-standing 200-nm-thick slab of silicon and the cell with the 210-nm-thick ITO of Fig. 3. As discussed before, larger thicknesses would be desirable for statistic treatment, but the central aspects of the following argument are also observed in the shown case with relatively thin films. First, we note that the dispersion diagram separates into two relatively independent waveguides; the effect is very clear for $s$ polarized light, but not yet fully pronounced in $p$-polarization for the shown thicknesses. Second, the absorption enhancement in a thick TCO "cladding" is obtained by counting the interactions between the light lines of air and the one of TCO, resulting in $4 n_{\mathrm{TCO}}^{2}$ (Ref. 13). Third, assessing the absorption enhancement in the silicon slab requires counting of all interactions between the light lines of air and silicon, resulting in

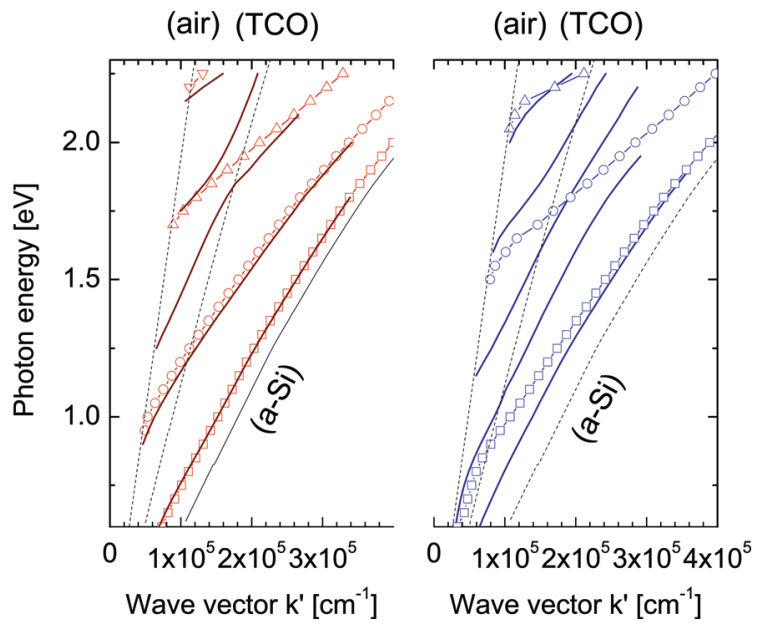

FIG. 11. (Color online) Dispersion diagram for the solar cell with $210 \mathrm{~nm}$ of ITO (cf. Fig. 3, lines) and for a 200-nm-thick slab of silicon delimited by air (symbols). As before, light lines are dashed, and the left- and right-hand panels denote $s$ - and $p$-polarization, respectively. 
$4 n_{\mathrm{Si}}^{2}$. Finally, we note that the interactions that enhance the absorption within the silicon layer of a device, take place between the light lines of TCO and silicon; the corresponding enhancement is therefore given by $4\left(n_{\mathrm{Si}}^{2}-n_{\mathrm{TCO}}^{2}\right)$. Note that this argument relies on two assumptions; the TCO contacts are thick, and their absorption is not negligible. If the latter were not the case, light that is guided in the cladding would eventually scatter into the silicon film or out of the device. Assuming approximate values of $n_{\mathrm{Si}} \approx 4$ and $n_{\mathrm{TCO}} \approx 2$, our conservative estimate of the enhancement is close to 50 , thus it still exceeds most experimental results. In periodic structures, peak absorption enhancement just below the appearance of first-order diffraction modes will be given by $F_{1 \mathrm{D}}=2 \pi\left(n_{\mathrm{Si}}\right.$ $\left.-n_{\mathrm{TCO}}\right)$ and $F_{2 \mathrm{D}}=4 \pi\left(n_{\mathrm{Si}}^{2}-n_{\mathrm{TCO}}^{2}\right)$ for $1 \mathrm{D}$ and $2 \mathrm{D}$ gratings, respectively.

\section{CONCLUSIONS}

We studied the absorption enhancement that can be achieved in thin film silicon solar cells by light scattering at textured interfaces. In the experimental part, we were able to relate the absorption enhancement in solar cells with periodic interface texture to a resonant excitation of waveguide modes. Combining 1D gratings and a variation of the incident angle allowed us to map out the modal structure independently for both polarization directions in a wide range of the Brillouin zone, and to compare it to a theoretical prediction of the dispersion relation for a realistic device structure. In the theoretical part, we applied our experimentally verified model of the mode structure to a recent prediction of the absorption enhancement based on a statistic distribution of energy among the modes of an idealized waveguide. Taking into account the fact that the propagation in a multilayer waveguide can be dominated by more than one of the constituent media, we propose to restrict the statistics only to those modes that represent the desired absorption in silicon. We conclude that conventional configurations of silicon thin film solar cells with thick and nonideal contacts may not attain the well-known Yablonovitch limit of $4 n_{\mathrm{Si}}^{2}$; instead, the upper limit of absorption enhancement is given by a more conservative value of $4\left(n_{\mathrm{Si}}^{2}-n_{\mathrm{TCO}}^{2}\right)$. For adequately designed $2 \mathrm{D}$ gratings, the limiting value is higher by a factor of $\pi$ in a small wavelength interval just above the grating period.

\section{ACKNOWLEDGMENTS}

We thankfully acknowledge Dr. Aïcha Hessler and Caroline Calderone for FIB cross sectioning and SEM imaging. This work was funded by the European Union within the project Si-Light (Contract No. 241277) and by the Swiss National Science Foundation (Contract No. 2000021_125177/1).
${ }^{1}$ D. E. Carlson and C. R. Wronski, Appl. Phys. Lett. 28(11), 671 (1976).

${ }^{2}$ H. W. Deckman, C. R. Wronski, H. Witzke, and E. Yablonovitch, Appl. Phys. Lett. 42, 968 (1983).

${ }^{3}$ H. Keppner, J. Meier, P. Torres, D. Fischer, and A. Shah, Appl. Phys. A 69, 169 (1999).

${ }^{4}$ O. Vetterl, F. Finger, R. Carius, P. Hapke, L. Houben, O. Kluth, A. Lambertz, A. Mück, B. Rech, and H. Wagner, Solar Energy Mater. Solar Cells 62, 97 (2000).

${ }^{5}$ M. Green, P. Basore, N. Chang, D. Clugston, R. Egan, R. Evans, D. Hogg, S. Jarnason, M. Keevers, and P. Lasswell, Solar Energy 77, 857 (2004).

${ }^{6}$ P. Campbell and M. Green, J. Appl. Phys. 62, 243 (2009).

${ }^{7}$ D. Redfield, Appl. Phys. Lett. 25, 647 (1974).

${ }^{8}$ E. Yablonovitch and G. D. Cody, IEEE Trans. Electron Devices 29, 300 (1982).

${ }^{9}$ P. Sheng, A. N. Bloch, and R. S. Stepleman, Appl. Phys. Lett. 43, 579 (1983).

${ }^{10}$ J. M. Gee, Proceedings of the 29th IEEE PVSC, New Orleans. LA, 2002, pp. 150-153.

${ }^{11}$ Z. Yu, A. Raman, and S. Fan, Opt. Express 18, A366 (2010).

${ }^{12}$ Z. Yu, A. Raman, and S. Fan, Proc. Natl. Acad. Sci. U.S.A. 107, 17491 (2010).

${ }^{13}$ M. A. Green, Prog. Photovoltaics (2010).

${ }^{14}$ F.-J. Haug, T. Söderström, O. Cubero, V. Terrazzoni-Daudrix, and C. Ballif, J. Appl. Phys. 104, 064509 (2008).

${ }^{15}$ C. Kothandaraman, T. Tonon, C. Huang, and A. E. Delahoy, Proceedings of the MRS Spring Meeting, MRS, San Francisco, 1991, pp. 475480 .

${ }^{16}$ S. Benagli, D. Borrello, E. Vallat-Sauvain, J. Meier, U. Kroll, J. Hoetzel, J. Bailat, J. Steinhauser, M. Marmelo, G. Monteduro, and L. Castens. Proceedings of the 24th European PVSEC, Hamburg, 2009, p. 3BO. 9.3.

${ }^{17}$ H. Okamoto, Y. Nitta, T. Adachi, and Y. Hamakawa, Surf. Sci. 86, 486 (1979).

${ }^{18}$ K. Söderström, F. Haug, J. Escarré, O. Cubero, and C. Ballif, Appl. Phys. Lett. 96, 213508 (2010)

${ }^{19}$ F. J. Haug, T. Söderström, O. Cubero, V. Terrazoni-Daudrix, and C. Ballif, J. Appl. Phys. 106, 044502 (2009).

${ }^{20}$ J. D. Jackson, Classical Electrodynamics, 3rd ed. (Wiley, New York, 1963).

${ }^{21}$ S. Shakir and A. Turner, Appl. Phys. A 29, 151 (1982).

${ }^{22}$ B. Götz, K. Hehl, W. Karthe, and B. Martin, IEEE J. Lightw. Technol. 11, 1447 (1993).

${ }^{23}$ I. Pockrand and H. Raether, Opt. Commun. 18, 395(1976).

${ }^{24}$ W. Burns and G. Hocker, Appl. Opt. 16, 2048 (1977).

${ }^{25}$ A. Otto, Z. Phys. 216, 398 (1968).

${ }^{26}$ P. K. Tien, R. Ulrich, and R. J. Martin, Appl. Phys. Lett. 14, 291 (1969).

${ }^{27}$ H. Raether, Opt. Commun. 42, 217 (1982).

${ }^{28}$ M. L. Dakss, L. Kuhn, P. F. Heidrich, and B. A. Scott, Appl. Phys. Lett. 16, 523 (1970).

${ }^{29}$ A. Naqavi, K. Söderström, F. J. Haug, V. Paeder, T. Scharf, H. P. Herzig, and C. Ballif, Opt. Express 19, 128 (2011).

${ }^{30}$ C. Haase and H. Stiebig, Prog. Photovoltaics 14, 629 (2006).

${ }^{31}$ C. Heine and R. H. Morf, Appl. Opt. 34, 2476 (1995).

${ }^{32}$ C. Rockstuhl, S. Fahr, K. Bittkau, T. Beckers, R. Carius, F. Haug, T. Söderström, C. Ballif, and F. Lederer, Opt. Express. 18, A335 (2010).

${ }^{33}$ H. Sakai, T. Yoshida, T. Hama, and Y. Ichikawa, Jpn. J. Appl. Phys., Part 1 29, 630 (1990).

${ }^{34}$ S. Schicho, D. Hrunski, R. van Aubel, and A. Gordijn, Prog. Photovoltaics 18, 83 (2010).

${ }^{35}$ M. Kambe, M. Fukawa, N. Taneda, Y. Yoshikawa, K. Sato, K. Ohki, S. Hiza, A. Yamada, and M. Konagai. Proceedings of the 3rd World PVSEC, Osaka, Japan, 2003, pp. 1812-1815. 